\title{
Feedforward Controller of Ill-Conditioned Hysteresis Using Singularity-Free Prandtl-Ishlinskii Model
}

\author{
U-Xuan Tan, Student Member, IEEE, Win Tun Latt, Student Member, IEEE, Cheng Yap Shee, \\ Cameron N. Riviere, Member, IEEE, and Wei Tech Ang, Member, IEEE
}

\begin{abstract}
Piezoelectric, magnetostrictive, and shape memory alloy actuators are gaining importance in high-frequency precision applications constrained by space. Their intrinsic hysteretic behavior makes control difficult. The Prandtl-Ishlinskii (PI) operator can model hysteresis well, albeit a major inadequacy: the inverse operator does not exist when the hysteretic curve gradient is not positive definite, i.e., ill condition occurs when slope is negative. An inevitable tradeoff between modeling accuracy and inversion stability exists. The hysteretic modeling improves with increasing number of play operators. But as the piecewise continuous interval of each operator reduces, the model tends to be ill-conditioned, especially at the turning points. Similar ill-conditioned situation arises when these actuators move heavy loads or operate at high frequency. This paper proposes an extended PI operator to map hysteresis to a domain where inversion is well behaved. The inverse weights are then evaluated to determine the inverse hysteresis model for the feedforward controller. For illustration purpose, a piezoelectric actuator is used.
\end{abstract}

Index Terms-Actuators, hysteresis, piezoelectric ceramics.

\section{INTRODUCTION}

$\mathbf{P}$ IEZOELECTRIC actuators are gaining an increasing importance in high-speed precision applications due to their high force, rapid response, compactness, and nonexistence of magnetic fields. The absence of backlash also allows nanometer positioning precision. Garmon et al. [1] utilized piezoelectric actuators in microsurgical tool, while Chang et al. [2] applied it in vibration compensation. However, the existence of nonlinear hysteresis complicates tracking control.

Current hysteresis modeling and compensation work can be categorized as: 1) microscopic theories; 2) electric charge control; 3) phase control; 4) closed-loop displacement control; and 5) linear control with feedforward inverse hysteresis model. Recent methods comprise a hybrid of the said methods.

Category (1) relates understanding of the microscopic structure to displacement. Landauer et al. [3] discussed the dependence of polarization, in barium titanate, on the field cycle rate.

Manuscript received June 20, 2008; revised August 26, 2008 and October 14, 2008. First published April 14, 2009; current version published September 2, 2009. Recommended by Technical Editor J. Ueda. This work was supported in part by the College of Engineering, Nanyang Technological University, in part by a Agency for Science, Technology and Research (A*STAR) Science and Engineering Research Council (SERC) grant, and in part by the National Institutes of Health (NIH) under Grant R01EB000526 and Grant R21EY016359.

U-X. Tan, W. T. Latt, C. Y. Shee, and W. T. Ang are with the School of Mechanical and Aerospace Engineering, Nanyang Technological University, Singapore 639798 (e-mail: tanu0002@ ntu.edu.sg; wintunlatt@ntu.edu.sg; cyshee@ntu.edu.sg; wtang@ntu.edu.sg).

C. N. Riviere is with the Robotics Institute, Carnegie Mellon University, Pittsburgh, PA 15213 USA (e-mail: camr@ri.cmu.edu).

Color versions of one or more of the figures in this paper are available online at http://ieeexplore.iee.org.

Digital Object Identifier 10.1109/TMECH.2008.2009936

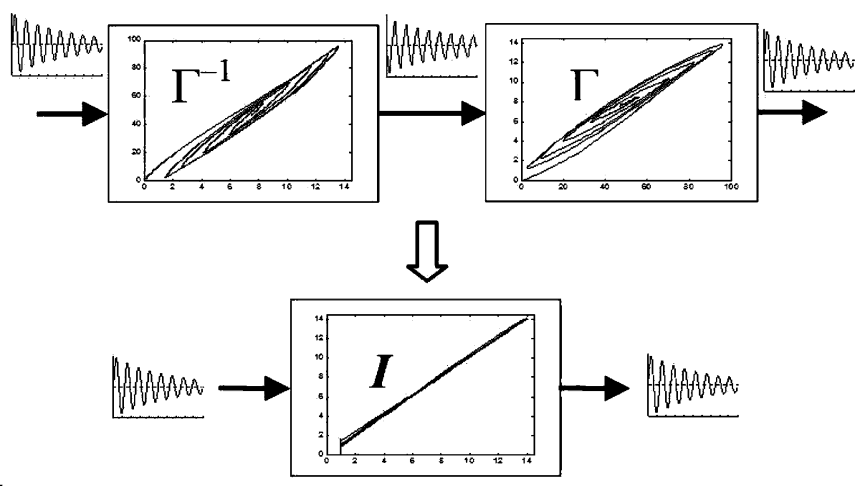

Fig. 1. Linearization of a hysteretic plant using the inverse feedforward controller.

Category (2) utilizes the knowledge that actuator displacement hysteresis to the applied voltage is about $15 \%$ while the displacement to induced charge is $2 \%$. This motivated Furutani et al. [4] to combine induced charge feedback with inverse transfer function compensation. Category (3) includes CruzHernandez and Hayward [5], [6] proposing phase control approach to design a compensator to reduce hysteresis. Category (4) comprises many different approaches. Some incorporated the inverse hysteresis model with a controller, while others proposed advance controllers utilizing neural network [7], [8], fuzzy logic [9], sliding mode [10], and $\mathrm{H}_{\infty}$ control [11].

Category (5), a phenomenological approach, obtains a mathematical model describing the hysteretic motion. This is commonly used as the study of the underlying physics of piezoelectric actuators requires expensive equipment. There are many attempts to derive various mathematical models that best describe the complex hysteretic motion. The inverse model is then used as a feedforward controller to linearize the hysteresis response (Fig. 1). The key idea of the inverse feedforward controller is to cascade the inverse hysteresis operator $\Gamma^{-1}$ with the actual plant's hysteresis $\Gamma$ to achieve an identity mapping between the desired actuator output and the actual actuator response.

The simplest method utilizes two sets of polynomials to model the forward and backward paths. However, this method will not work when the turning point location changes as the polynomials will not be continuous. Sun et al. [12] proposed a new mathematical model by modifying the polynomials.

$\mathrm{Hu}$ and Ben-Mrad [13], [14] and Song et al. [15] employed the popular Preisach model, while Goldfarb and Celanovic [16] used Maxwells model. Tao [17] used the hysteron model. The rest are variations from the classical models. 




Fig. 2. Ill-conditioned hysteresis.

Another model is the Prandtl-Ishlinskii (PI) model. Kuhnen [18] and Kuhnen and Janocha [19] demonstrated that the PI operator (mathematically less complex with its inverse computed analytically) is suited for real-time applications. But the operating frequency must not be too high as the hysteresis nonlinearity will become severe. Similarly, the PI model cannot function as a feedforward controller when the largest displacement does not occur at the highest input signal (Fig. 2) as singularity occurs in the inverse. Most systems can be explained using a springmass-damper system. At high velocity, the actuator has a high momentum at the turning point, especially if a rapid change is made. The large momentum keeps the system in motion resulting in the convex curve. This is also applicable for large loads. The PI model cannot be used as a feedforward controller for such cases as the inverse operator does not exist when the hysteretic curve gradient is not positive definite, i.e., singularity occurs. Thus, the author proposed to model the hysteresis in a different domain when such situations are encountered.

This paper (an in-depth version of [20]) presents a solution to overcome the ill-conditioned problem. Section II reviews the PI hysteresis model. Section III presents the proposed transformation of the ill-conditioned hysteresis onto another domain. Section IV gives the experimental results. Sections V and VI cover the discussion and conclusions, respectively.

\section{Hysteresis Mathematical Model}

This section briefly introduces the PI model proposed by Kuhnen [18] and Kuhnen and Janocha [19], with a solution by Ang et al. [21] to account for the one-sided characteristic as piezoelectric actuators usually operate on the positive region.

\section{A. Prandtl-Ishlinskii}

The play operator in the PI hysteresis model, commonly used to model the backlash between gears, is defined by

$$
\begin{aligned}
y(t) & =H_{r}\left[x, y_{0}\right](t) \\
& =\max \{x(t)-r, \min [x(t)+r, y(t-T)]\}
\end{aligned}
$$

where $x$ is the control input, $y$ is the actuator response, $r$ is the control input threshold value or the magnitude of the backlash, and $T$ is the sampling period. Initial condition of (1) is given by

$$
y(0)=\max \left\{x(0)-r, \min \left[x(0)+r, y_{0}\right]\right\}
$$

where $y_{0}$ is a real number that is usually initialized to 0 . To change the gradient, a weight $w_{h}$ is premultiplied to $H_{r}$. By summing a number of such operators with different threshold values and weights, a hysteresis model is obtained

$$
y(t)=\vec{w}_{h}^{T} \vec{H}_{r}\left[x, \vec{y}_{0}\right](t)
$$

where weight vector $\vec{w}_{h}^{T}=\left[w_{h_{0}} \cdots w_{h_{n}}\right]$ and $\vec{H}_{r}\left[x(t), \vec{y}_{0}\right]=$ $\left[H_{r_{0}}\left[x(t), y_{0_{0}}\right] \cdots H_{r_{n}}\left[x(t), y_{0_{n}}\right]\right]^{T}$ with the threshold vector $\vec{r}=\left[r_{0} \cdots r_{n}\right]^{T}$, where $r_{n}>\cdots>r_{0}, r_{0}=0$, and the initial state vector $\vec{y}_{0}=\left[y_{0_{0}} \cdots y_{0_{n}}\right]^{T}$. Unlike the Preisach model, which behaves like a number of steps, the PI operator is mathematically simpler and also a better mathematical model as it is a first-order gradient. To account for the one-sided characteristics of the actuators, Ang et al. [21] proposed setting the value of $r_{n}$ to half of the maximum control input.

The nature of PI operator is symmetrical about the center of the loop, but this is not true in the phenomena observation. To overcome this restriction, Ang et al. [21] and Kuhnen [18] proposed using a one-sided dead zone operator

$$
\begin{aligned}
S_{d}[y](t) & = \begin{cases}\max \{y(t)-d, 0\}, & d>0 \\
y(t), & d=0\end{cases} \\
z(t) & =\vec{w}_{s}^{T} \vec{S}_{d}[y(t)]
\end{aligned}
$$

where weight vector $\quad \vec{w}_{s}^{T}=\left[w_{s_{0}} \cdots w_{s_{m}}\right], \vec{S}_{d}[y(t)]=$ $\left[S_{d_{0}}[y(t)] \cdots S_{d_{m}}[y(t)]\right]^{T}, d$ is the threshold value in the dead zone operator, and $z$ is the actuator's displacement response. The general idea of the one-sided dead zone operators is to bend the graph and make it not symmetrical.

\section{B. Parameter Identification}

A phenomenological approach is like memorizing the path. Experimental values are gathered to obtain the hysteresis model. After obtaining the experimental data, least-square minimization of the error function $E[x, y]$ is performed to the weights $\overrightarrow{w_{h}}$ and $\overrightarrow{w_{s}}$ using

$$
E[x, y]\left(\vec{w}_{h}, t\right)=\vec{w}_{h}^{T} \vec{H}_{r}\left[x, \vec{y}_{0}\right](t)-\vec{w}_{s^{\prime}}^{T} \vec{S}_{d^{\prime}}[z(t)]
$$

where $\vec{w}_{h^{\prime}}^{T}$ and $\vec{w}_{s^{\prime}}^{T}$ are the inverse parameters of $\vec{w}_{h}^{T}$ and $\vec{w}_{s}^{T}$, respectively, and $\vec{H}_{r^{\prime}}$ and $\vec{S}_{d^{\prime}}$ are the $\vec{H}_{r}$ and $\vec{S}_{d}$ operators with the inverse of $\vec{r}$ and $\vec{d}$ as the input, respectively. The inverse parameters can be obtained by (9). This error function, obtained from [18], is recommended over position error because the error will be linearly dependent on the weights.

\section{Inverse Model}

The inverse PI model is commonly expressed by stop operators. Kuhnen [18] showed that the inverse model can be expressed by play operators too. Thus, the inverse model is expressed as

$$
\Gamma^{-1}[z(t)]=\vec{w}_{h^{\prime}}^{T} \vec{H}_{r^{\prime}}\left[\vec{w}_{s^{\prime}}^{T} \vec{S}_{d^{\prime}}[z],{\overrightarrow{y^{\prime}}}_{0}\right](t) .
$$

The graphical representation of finding the inverse is to find the mathematical model that describes the hysteresis path after a reflection along the $45^{\circ}$ line. Using the inverse as a feedforward 


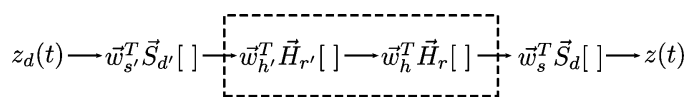

Fig. 3. Signal flow of the inverse feedforward controller.

controller gives a linear response, as shown in Fig. 1. Mathematically speaking,

$$
\begin{aligned}
z(t) & =\Gamma\left[\Gamma^{-1}[x(t)]\right] \\
& =x(t) .
\end{aligned}
$$

The inverse model parameters can be calculated by

$$
\begin{gathered}
w_{h_{0}}^{\prime}=\frac{1}{w_{h_{0}}}, \quad w_{h_{i}}^{\prime}=\frac{-w_{h i}}{\left(\sum_{j=0}^{i} w_{h_{j}}\right)\left(\sum_{j=0}^{i-1} w_{h_{j}}\right)}, \\
r_{i}^{\prime}=\sum_{j=0}^{i} w_{h_{j}}\left(r_{i}-r_{j}\right), \quad y_{0_{i}}^{\prime}=\sum_{j=0}^{i} w_{h_{j}} y_{0_{i}}+\sum_{j=i+1}^{n} w_{h_{j}} y_{0_{j}} \\
w_{s_{0}}^{\prime}=\frac{1}{w_{s_{0}}}, \quad w_{s_{i}}^{\prime}=\frac{r_{0}=0, \quad i=0, \ldots, n}{\left(\sum_{j=0}^{i} w_{s_{j}}\right)\left(\sum_{j=0}^{i-1} w_{s_{j}}\right)}, \\
d_{i}^{\prime}=\sum_{j=0}^{i} w_{s_{j}}\left(d_{i}-d_{j}\right), \quad i=0, \ldots, m .
\end{gathered}
$$

Fig. 3 shows the signal flow diagram of the inverse feedforward controller. The desired displacement $z(t)$ is first passed through the inverse dead zone operators, followed by the inverse play operators to obtain the required voltage. This voltage is then passed through the hysteretic plant, which is modeled as by the play operators followed by dead zone operators. However, there is a limitation when finding the inverse parameters of the play operators. Linearization of the boxed area in Fig. 3 will fail. The equations to find the inverse parameters of the play operators (9) fail when negative gradient is encountered. The inverse parameters of the play operators were derived by Kuhnen [18] under the consideration of the following linear inequality constraints $[18$, eq. (8)]:

$$
\mathbf{U}_{H} \cdot \vec{w}_{h}-\mathbf{u}_{H} \leq \mathbf{0}
$$

where

$$
\mathbf{U}_{H}=\left(\begin{array}{cccc}
-1 & 0 & \cdots & 0 \\
0 & -1 & \cdots & 0 \\
\vdots & \vdots & \vdots & \vdots \\
0 & 0 & \cdots & -1
\end{array}\right), \quad \mathbf{u}_{H}=\left(\begin{array}{c}
-\varepsilon \\
0 \\
\vdots \\
0
\end{array}\right)
$$

and $\varepsilon$ is a very small positive number. This linear inequality assumes the slope to be positive, and the inverse equation is not applicable when negative slope is encountered. An example is illustrated here. Given the weights $w_{h}^{T}=$ [ $\left.\begin{array}{llllll}-0.2 & 0.1 & 0.2 & 0.2 & 0.2 & 0.2\end{array}\right]$ and $\vec{r}=\left[\begin{array}{lllll}0 & 1 & 2 & 3 & 4\end{array}\right]$ for an application where the amplitude of the periodic input voltage is $10 \mathrm{~V}$, the loading curve obtained is shown in Fig. 4.

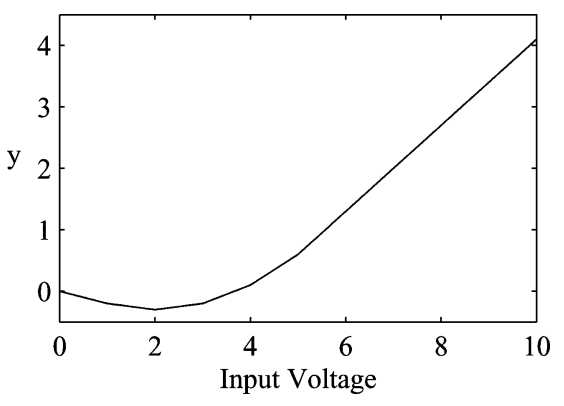

Fig. 4. Loading curve of an ill-conditioned hysteresis involving negative gradient.

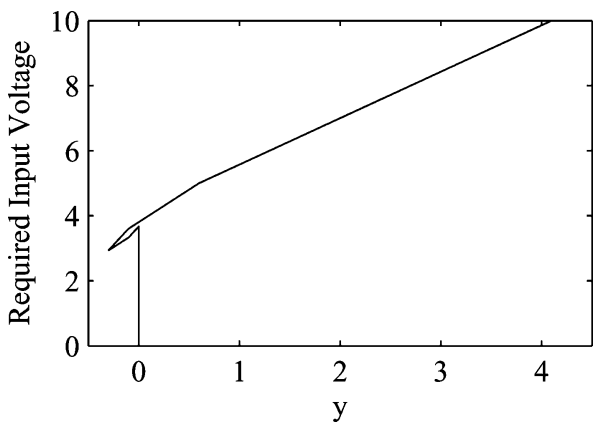

Fig. 5. Inverse loading curve of an ill-conditioned hysteresis to illustrate failure of the PI inverse operator when negative gradient is encountered.

Applying (9) to get the inverse PI parameters, the parameters obtained are $\vec{w}_{h}^{T}=[-5-520-6.6667-1.333-$ $0.5714]$ and $\vec{r}^{\prime}=[0-0.2-0.3-0.20 .10 .6]$. Fig. 5 illustrates the inverse curve that is obtained using (9). It can be seen that the inverse curve obtained is not a reflection of the loading curve along the $45^{\circ}$ line. This simple example clearly illustrates that (9) fail to find the correct inverse weights when negative gradient is encountered. Zero gradient is not demonstrated as it is clear that the reciprocal of 0 is a singular point.

\section{Modeling IN A DifFerent Domain}

This section proposes a method for dealing with situations when negative gradient is encountered and the inverse of the PI operator cannot be evaluated properly. This section begins with the intuition, followed by the method and simulation.

\section{A. Intuition}

With the singularity problem, the idea to model the hysteresis in an alternative domain, which is always well conditioned, is proposed. A transformation is used to map $y$ to $y_{1}$, which has no singular points, as shown in Fig. 6. The inverse model can now be obtained in the new domain. If the relationship between $y$ and $y_{1}$ is known, the appropriate input voltages can be obtained using the inverse model found in the new domain, as shown in Fig. 7.

The desired value displacement $y$ is first passed through the transformation function to obtain the corresponding new domain $y_{1}$ value. This $y_{1}$ value is then passed through the inverse model obtained in the new domain to get the appropriate input voltages. 


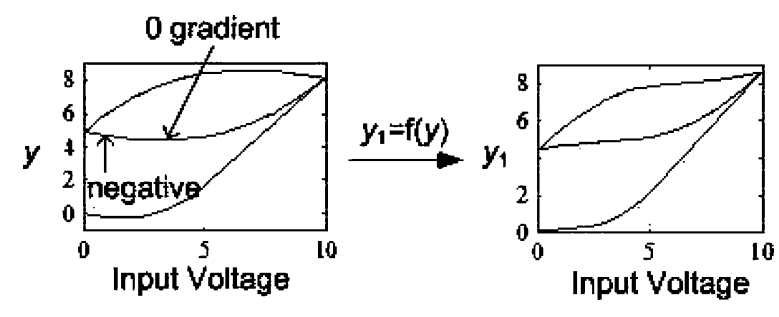

Fig. 6. Transformation.

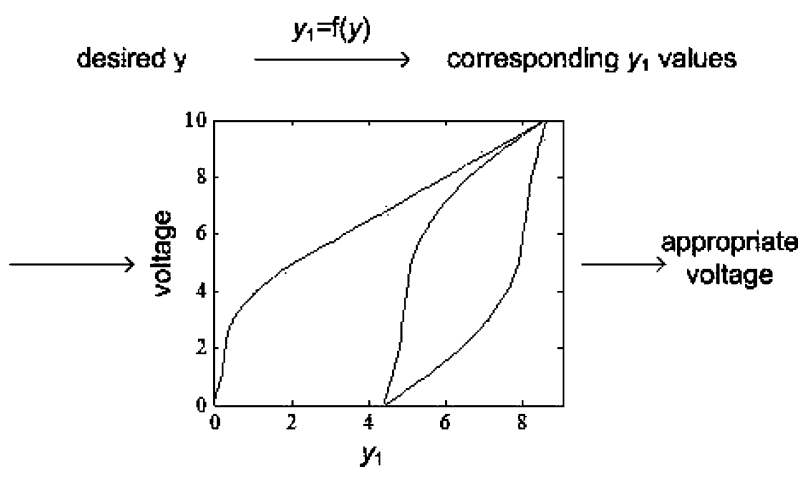

Fig. 7. Method to obtain appropriate input voltage.

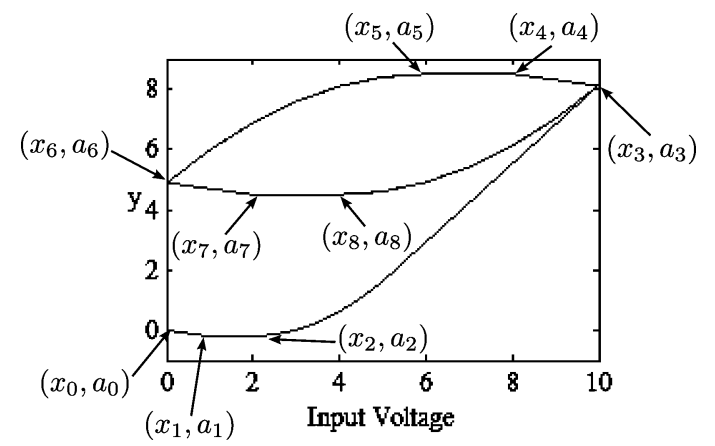

Fig. 8. Graph of an ill-conditioned hysteresis with points $x_{0}$ to $x_{8}$ labeled.

\section{B. Solution}

Although the inverse fails, the PI model can still describe the phenomena path like Fig. 2. The weights of the play operators for the ill-conditioned hysteresis can be obtained as shown in Section II by performing least-square error to (6). To obtain the relationship between the two domains ( $y$ and $y_{1}$ ), nine points $\left(x_{0}-x_{8}\right)$ are identified, as labeled in Fig. 8.

The region between $x_{0}$ and $x_{1}$ is for the negative gradient while 0 gradient is between $x_{1}$ and $x_{2}$. In the hysteresis loop, region between $x_{3}$ to $x_{4}$ and $x_{6}$ to $x_{7}$ has negative gradient, while $x_{4}$ to $x_{5}$ and $x_{7}$ to $x_{8}$ contain gradient value 0 . The labeled points $x_{0}$ to $x_{8}$ can be calculated using

$$
\begin{aligned}
& x_{0}=0 \\
& \text { If } \sum_{j=1}^{i} w_{h_{j}}=0 \text { exists, } \quad x_{1}=r_{i}, \quad x_{2}=r_{i+1} \\
& \text { otherwise } x_{1}=x_{2}=r_{\max }, \quad \text { where } \sum_{j=1}^{\max } w_{h_{j}}>0
\end{aligned}
$$



Fig. 9. Relationship between $y_{1}$ (new domain) and $y$.

$$
\begin{aligned}
& x_{3}=2 r_{\max } \\
& x_{4}=x_{3}-2 x_{1} \\
& x_{5}=x_{3}-2 x_{2} \\
& x_{6}=0 \\
& x_{7}=x_{6}+2 x_{1} \\
& x_{8}=x_{6}+2 x_{2} .
\end{aligned}
$$

Next, a transformation function to map $y$ domain onto a new domain $y_{1}$, which has no singular point, has to be proposed. The transformation function should be the one that is able to obtain the relationship between $y$ and $y_{1}$ easily. This paper proposed to keep the slope's amplitude of $y_{1}$ against voltage to be the same as $y$ against voltage, except for region where the gradient is near 0 . Negative slopes are reflected along the horizontal axis to obtain a positive, but equal amplitude slope. In this way, the gradient of the relationship between $y_{1}$ versus $y$ is either 1 , -1 , or infinite. This makes the formation of the relationship between the two domains easy to establish. Thus, the following transformation function to obtain the new weights is proposed

$w_{2 h_{i}}= \begin{cases}-\sum_{j=1}^{i} w_{h_{j}}-\sum_{j=1}^{i-1} w_{2 h_{j}}, & \sum_{j=1}^{i} w_{h_{j}}<0 \\ c-\sum_{j=1}^{i-1} w_{2 h_{j}}, & \sum_{j=1}^{i} w_{h_{j}}=0 \\ \sum_{j=1}^{i} w_{h_{j}}-\sum_{j=1}^{i-1} w_{2 h_{j}}, & \sum_{j=1}^{i} w_{h_{j}}>0\end{cases}$

where $c$ is a positive nonzero constant. The objective of this transformation is to ensure that the gradient of the hysteresis in the new domain is positive throughout. The first expression will reflect all the negative gradients to become positive. The second expression will force a 0 gradient to become a positive nonzero number. The third expression will allow the positive gradient to remain the same value. In addition, the new domain is of a PI type.

Note that with this transformation, the amplitude of the gradient at all points is maintained. This makes the relationship between $y$ and $y_{1}$ easy to express. Fig. 9 shows the relationship between the two domains. The constants $a_{i}$ and $b_{i}$ are the corresponding $y$ and $y_{1}$ values, respectively, to input voltage $x_{i}$. 
TABLE I

RELATION BETWEEN THE Two DOMAINS

\begin{tabular}{lc}
\hline \hline$y$ value & corresponding $y_{1}$ value \\
$a_{0}$ to $a_{1}$ & $y_{1}=-y$ \\
$a_{1}$ to $a_{2}$ & $y_{1}=b_{2}$, any value between $b_{1}$ and $b_{2}$ \\
$a_{2}$ to $a_{3}$ & $y_{1}=y+b_{2}-a_{2}$ \\
$a_{3}$ to $a_{4}$ & $y_{1}=-y+b_{3}+a_{3}$ \\
$a_{4}$ to $a_{5}$ & $y_{1}=b_{5}$, any value between $b_{4}$ and $b_{5}$ \\
$a_{5}$ to $a_{6}$ & $y_{1}=y+b_{5}-a_{5}$ \\
$a_{6}$ to $a_{7}$ & $y_{1}=-y+b_{6}+a_{6}$ \\
$a_{7}$ to $a_{8}$ & $y_{1}=b_{8}$, any value between $b_{7}$ and $b_{8}$ \\
\hline
\end{tabular}

As the amplitude of the slope is maintained, all the gradients of the lines relating $y_{1}$ and $y$ are $1,-1$, or infinite. Points $b_{0}$ to $b_{8}$ can be obtained using

$$
\begin{aligned}
& b_{0}=0 \\
& b_{1}=-a_{1} \\
& b_{2}=b_{1}+c \times\left[x_{2}-x_{1}\right] \\
& b_{3}=b_{2}+\left[a_{3}-a_{2}\right] \\
& b_{4}=b_{3}-\left[a_{4}-a_{3}\right] \\
& b_{5}=b_{4}-2 c \times\left[x_{2}-x_{1}\right] \\
& b_{6}=b_{5}+\left[a_{6}-a_{5}\right] \\
& b_{7}=b_{6}-\left[a_{7}-a_{6}\right] \\
& b_{8}=b_{7}+2 c \times\left[x_{2}-x_{1}\right] .
\end{aligned}
$$

With these points, the relation between $y_{1}$ and $y$ can be expressed as in Table I.

\section{Generation of the Inverse Model}

This section demonstrates that the inverse model obtained is indeed the reflection along the $45^{\circ}$ line. The $y$ value is first obtained from the desired displacement $z$ by passing it through the inverse dead zone operator. The corresponding $y_{1}$ value is then obtained from $y$ using Table I. The $y_{1}$ value is then passed through the inverse model obtained in the new domain to get the appropriate input voltage. The final inverse graph is plotted with the ill-conditioned hysteresis in Fig. 10. As shown in Fig. 10, the final inverse graph (dotted) is a reflection of the hysteresis graph along the $45^{\circ}$ line. This section has clearly illustrated the ability of the transformation function to obtain the inverse of the illconditioned hysteresis curve. The transformation function has no effect on well-conditioned hysteresis as $x_{2}=x_{1}=x_{0}=0$.

\section{Simulation}

Next, a feedforward linearization simulation is performed on an ill-conditioned hysteretic plant with the following param-


$\left[\begin{array}{lllllll}-1.6030 & 2.1478 & 4.3923 & -3.2764 & 0.7664 & 0.1845 & 0.2283\end{array}\right.$

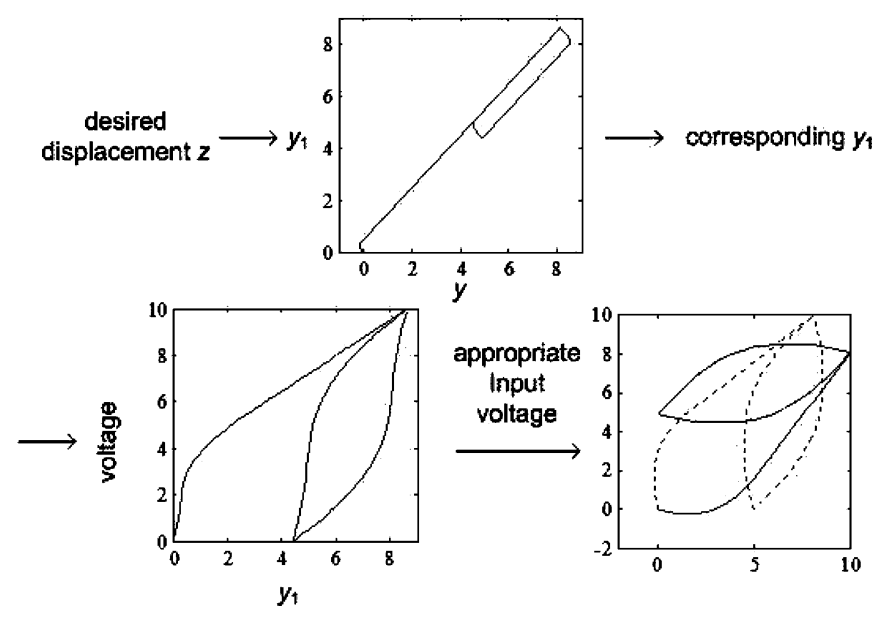

Fig. 10. Simulation to show that inverse after mapping is the reflection along the $45^{\circ}$ line.
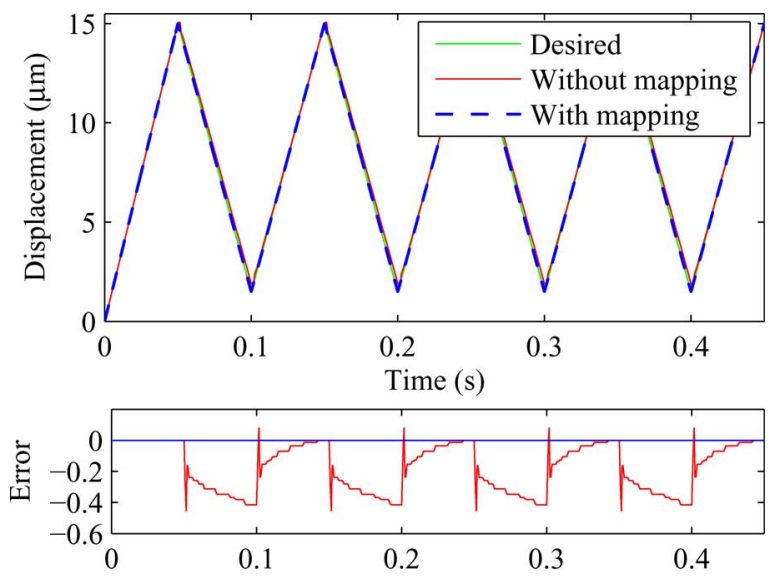

Fig. 11. Simulation result of the feedforward linearization (output result).

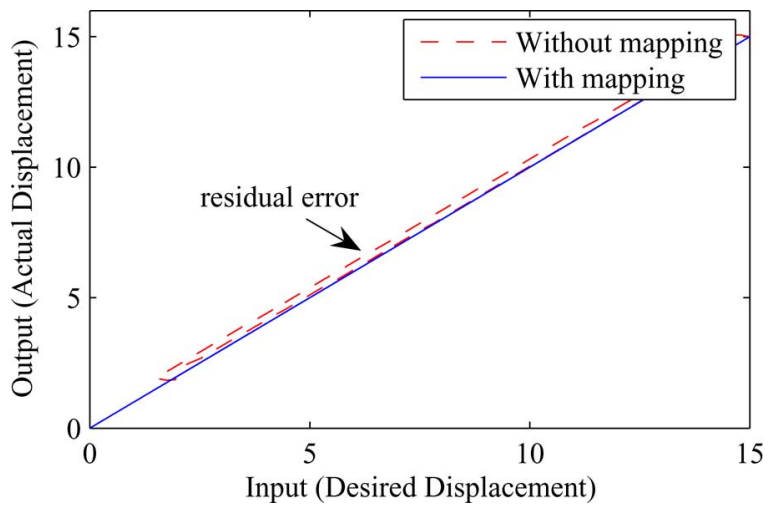

Fig. 12. Simulation result of the feedforward linearization (input-output linearization effect).

$0.32220 .35150 .21760 .1358-0.3989], \vec{d}=\left[\begin{array}{lll}0 & 8 & 12\end{array}\right], \quad$ and $\vec{w}_{s}^{T}=[0.48538-0.02548-0.01384]$. The simulation is performed with and without the proposed method, and the results are compared. Fig. 11 shows the output result, while Fig. 12 shows the input-output linearization effect. Residual error can be clearly seen if mapping is not used. 


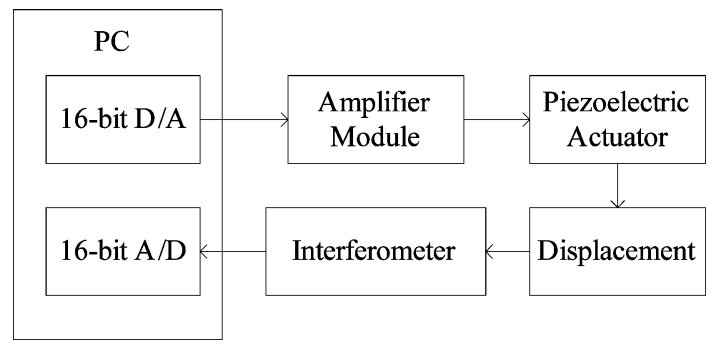

Fig. 13. Experimental architecture.

\section{EXPERIMENTAL RESULTS}

In this section, the behavior of the PICMA P885.50 (a multilayered piezoceramic with a stroke length of $15 \mu \mathrm{m}$ at $100 \mathrm{~V}$ ) from Physik Instrumente is modeled using the rate-dependent model proposed by Ang et al. [21]. The rate-dependent model in [21] basically calculates the weights according to velocity. With these weights, the inverse model is then obtained via the method discussed. A brief description of the experiment set is first given, followed by several experiments to show the improvements. The first set of experiments is $8 \mathrm{~Hz}$ triangular wave, followed by varying-amplitude linear motion with varying velocity to demonstrate the capability to model the rate-dependent model. The last experiment is a varying frequency with varying amplitude sinusoidal wave.

\section{A. Experimental Setup}

As seen from Fig. 13, a 16-bit D/A card is used to generate the voltage, which is then passed through the amplifier (gain is 10). Given the voltage, the actuator will deform and the interferometer (Philtec RC12 with a resolution of $0.08 \mu \mathrm{m}$ ) will detect the displacement and convert to analog voltage signal that is read in via a 16-bit A/D card.

\section{B. Experimental Result}

The actuator is first modeled. The same model is being used for both with and without mapping. The first experiment's desired displacement is a triangular wave with the velocity high enough for the first weight to enter into the negative region, while the second experiment's desired displacement is nonperiodic linear motion. Figs. 14-16 show the results of the triangular wave, while Figs. 17 and 18 show the outcome of the nonperiodic motion. Table II summarizes the result.

From Figs. 14 and 16, it can be clearly seen that the error has a general shape of a square wave. It has a general offset of overshoot when the desired displacement is increasing and undershoot when decreasing. With mapping, these overshoots or undershoots are removed. It can be clearly seen that the error in Fig. 14 (without mapping) is higher than the error in Fig. 15 (with mapping), and the rms error is greatly reduced by $40.1 \%$. This also proved that the error is due to the ill condition and not the hysteresis model.

Similar findings were obtained with nonperiodic linear motion. Figs. 17 and 18 show the result of without and with mapping, respectively. As seen from Fig. 17, like Fig. 14, there is

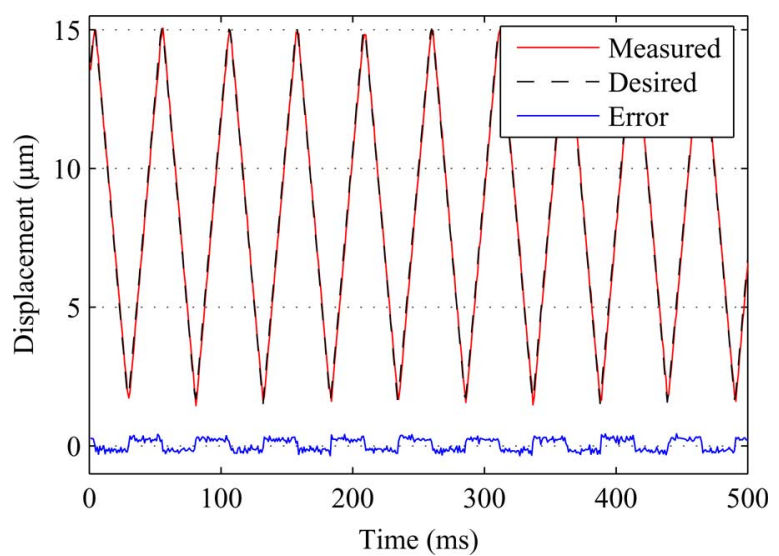

Fig. 14. Triangular wave without mapping.

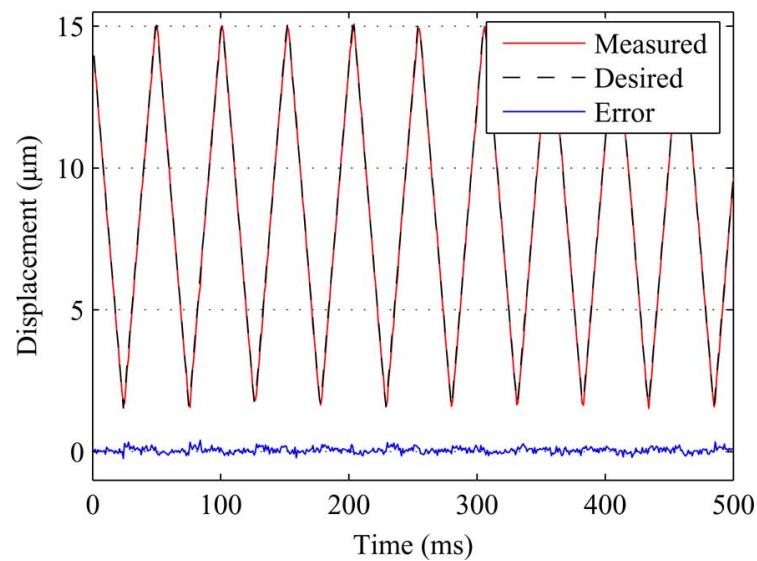

Fig. 15. Triangular wave with mapping.
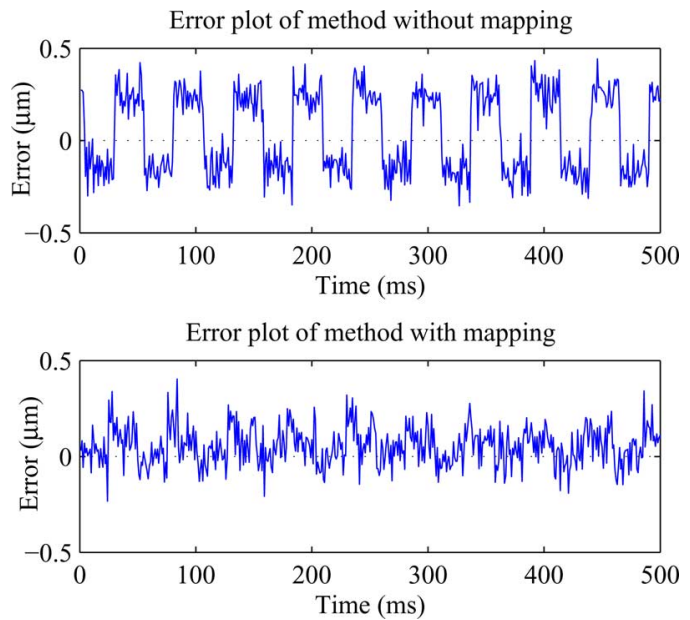

Fig. 16. Exploded view of error of triangular wave without and with mapping, respectively.

also a constant overshoot or undershoot in the error depending on the direction of actuation. With mapping, the offsets are removed and the rms error for this particular motion (calculated over the $200 \mathrm{~ms}$ ) is reduced by $41.4 \%$.

Fig. 19 is an experiment to show that the model is also valid for nonperiodic varying sinusoidal waves and the rms error obtained 



Fig. 17. Nonperiodic linear motion without mapping.
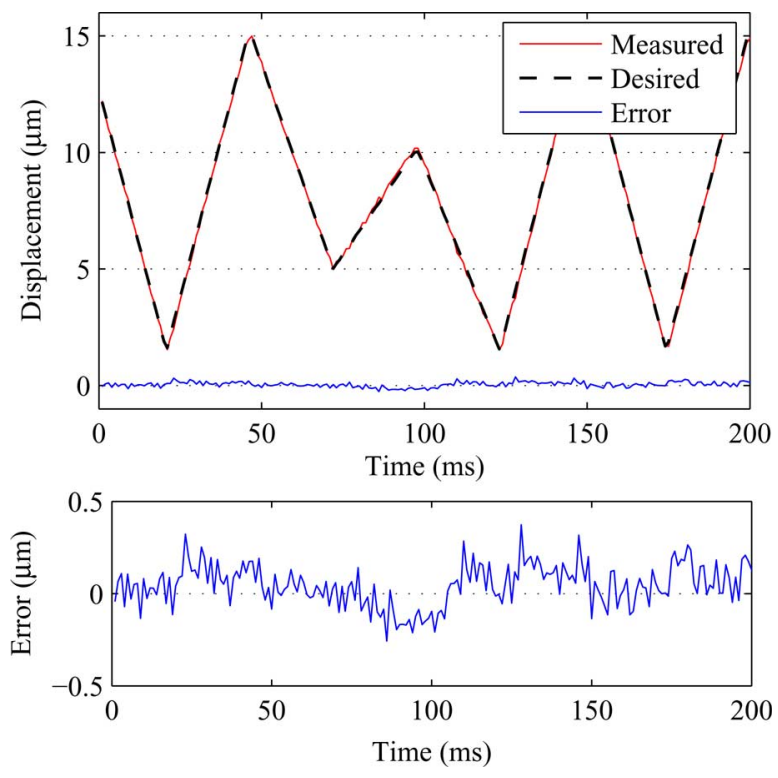

Fig. 18. Nonperiodic linear motion with mapping.

TABLE II

EXPERIMENTAL RESULTS

\begin{tabular}{ccccc}
\hline \hline & \multicolumn{2}{c}{ Triangular Motion } & \multicolumn{2}{c}{$\begin{array}{c}\text { Non-Periodic Linear } \\
\text { Motion }\end{array}$} \\
\hline & Without & With & Without & With \\
rmse $(\mu \mathrm{m})$ & 0.2580 & 0.1544 & 0.2267 & 0.1328 \\
rms error reduction & $40.1 \%$ & \multicolumn{2}{c}{$41.4 \%$} \\
max. error $(\mu \mathrm{m})$ & 0.5478 & 0.4222 & 0.4959 & 0.3473
\end{tabular}

This table summarizes the experimental results. A comparison between without mapping and with mapping can be made from this table.
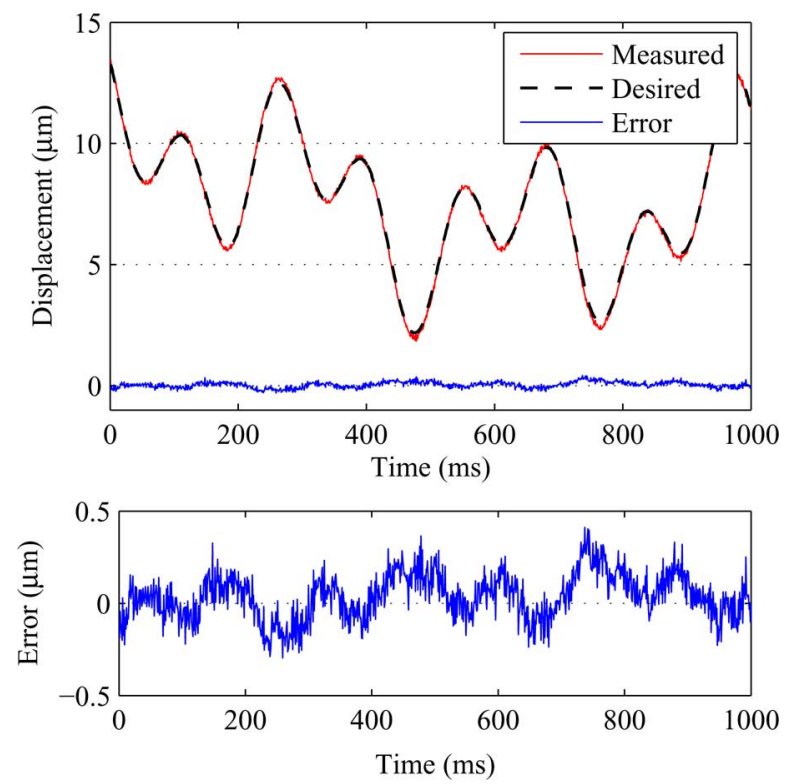

Fig. 19. Superposition of a few different frequencies of sinusoidal waves.

is $0.14436 \mu \mathrm{m}$. These figures demonstrate that the model is able to handle nonperiodic motion.

\section{DISCUSSION}

The PI model is the proposed method because models like Preisach can model only continuously increasing gradient, while PI is able to model hysteresis like Fig. 2. In addition, PI is the preferred method because the gradient of each segment can be obtained easily without any numerical differentiation needed. The inverse PI model can be computed easily too.

In this singularity-free PI model, the formulas formed are based on one assumption. The assumption made is the existence of only one maximum and one minimum turning point. Some modifications will have to be made if there are two or more maximum or minimum turning points. However, there is not much need to bother about this factor as very few systems have such irregular hysteresis. Even if such situation arises, the reader can use the proposed method with similar modification to account for the ill-shaped hysteresis.

Although the PI model is being used in this paper, the idea of transformation can be extended to other models, and the authors intend to do that in the near future because there are different advantages in each model to suit different situations.

From Fig. 16, it can be clearly seen that the error has a general shape of a square wave. It has a general offset of overshoot when the desired displacement is increasing and an offset of undershoot when the displacement is decreasing. The cause of this error is the singularity problem in the inverse PI hysteresis model. By mapping the hysteresis model onto a singularity-free domain, the rms error was decreased by $40.1 \%$ (from 0.2580 to $0.1544 \mu \mathrm{m})$.

There is, however, one negative factor. When the desired motion is a varying-frequency sinusoidal wave (not illconditioned), the method will actually increase the rms error 
by approximately $0.03-0.05 \mu \mathrm{m}$. The small error is due to the constantly changing relationship between $y$ and $y_{1}$. This error surfaces when the rate of change of velocity is high. This is due to the rate-dependent model used. A change of velocity in the model results in change in the weights of the play operators. As seen in Fig. 19, the error is highest at the turning point as this is also where the highest acceleration is. One possible method to reduce the error is to increase the sampling rate to make the change in weights between samples smaller. Nevertheless, it is more desirable to achieve a singularity-free model and inquire $0.05 \mu \mathrm{m}$ of error than to have a controller that will be unstable when the singularity situation is met.

Although the application here is with piezoelectric actuators, the singularity-free hysteresis model that the authors came out with is a general model and is applicable to any hysteresis materials (applicable to most smart materials) or systems.

\section{CONCLUSION}

The main contribution of this paper was to extend the PI model to include ill-conditioned hysteresis in the feedforward controller. The classical PI model is unable to find the inverse of the hysteresis phenomenon when the largest displacement does not occur at the highest input signal. Thus, a transformation to map the ill-conditioned hysteresis graph onto a betterconditioned domain was demonstrated in this paper. Simulations and experiments were also conducted.

\section{REFERENCES}

[1] F. A. Garmon, W. T. Ang, P. K. Khosla, and C. N. Riviere, "Rate-dependent inverse hysteresis feedforward controller for microsurgical tool," in Proc. 25th Int. Conf. IEEE EMBS, Cancun, Mexico, 2003, pp. 3415-3418.

[2] T. N. Chang, R. Kwadzogah, and R. J. Caudill, "Vibration control of linear robots using a piezoelectric actuator," IEEE/ASME Trans. Mechatronics, vol. 8, no. 4, pp. 439-445, Dec. 2003.

[3] R. Landauer, D. R. Young, and M. E. Drougard, "Polarization reversal in the barium titanate hysteresis loop," J. Appl. Phys., vol. 27, no. 71, pp. 752-758, 1956.

[4] K. Furutani, M. Urushibata, and N. Mohri, "Improvement of control method for piezoelectric actuator by combining induced charge feedback with inverse transfer function compensation," in Proc. IEEE Int. Conf. Robot. Autom., Leuven, Belgium, May 1998, pp. 1504-1509.

[5] J. M. Cruz-Hernandez and V. Hayward, "Phase control approach to hysteresis reduction," IEEE Trans. Control Syst. Technol., vol. 9, no. 1, pp. 17-26, Jan. 2001.

[6] J. M. Cruz-Hernandez and V. Hayward, "Reduction of major and minor hysteresis loops in a piezoelectric actuator," in Proc. 37th IEEE Conf. Decis. Control, Tampa, FL, 1998, pp. 4320-4325.

[7] S.-S. Ku, U. Pinsopon, S. Cetinkunt, and S. I. Nakajima, "Design, fabrication, and real-time neural network control of a three-degrees-of-freedom nanopositioner," IEEE/ASME Trans. Mechatronics, vol. 5, no. 3, pp. 273280, Sep. 2000

[8] C.-L. Hwang and C. Jan, "A reinforcement discrete neuro-adaptive control for unknown piezoelectric actuator systems with dominant hysteresis," IEEE Trans. Neural Netw., vol. 14, no. 1, pp. 66-78, Jan. 2003.

[9] Y. Stepanenko and C.-Y. Su, "Intelligent control of piezoelectric actuators," in Proc. 37th IEEE Conf. Decis. Control, Tampa, FL, Dec.1998, pp. 4234-4239.

[10] K. Abidi, A. Sabanovic, and S. Yesilyurt, "Sliding mode control based disturbance compensation and external force estimation for a piezoelectric actuator," in Proc. 8th IEEE Int. Workshop Adv. Motion Control, Mar. 2004, pp. 529-534.

[11] B. M. Chen, T. H. Lee, C. C. Hang, Y. Guo, and S. Weerasmriya, "An H $\infty$ almost disturbance decoupling robust controller design for a piezoelectric bimorph actuator with hysteresis," IEEE Trans. Control Syst. Technol., vol. 7, no. 2, pp. 160-174, Mar. 1999.
[12] L. Sun, C. Ru, W. Rong, L. Chen, and M. Kong, "Tracking control of piezoelectric actuator based on a new mathematical model," J. Micromech. Microeng., vol. 14, no. 11, pp. 1439-1444, 2004.

[13] H. Hu and R. Ben-Mrad, "Enhancement of tracking ability in piezoceramic actuators subject to dynamic excitation conditions," IEEE/ASME Trans. Mechatronics, vol. 10, no. 2, pp. 230-239, Apr. 2005.

[14] H. Hu and R. Ben-Mrad, "A model for voltage-to-displacement dynamics in piezoceramic actuators subject to dynamic-voltage excitations," IEEE/ASME Trans. Mechatronics, vol. 7, no. 4, pp. 479-489, Dec. 2002.

[15] G. Song, J. Zhao, X. Zhou, and J. A. D. Abreu-Garcia, "Tracking control of a piezoceramic actuator with hysteresis compensation using inverse Preisach method," IEEE/ASME Trans. Mechatronics, vol. 10, no. 2, pp. 198-209, Apr. 2005.

[16] M. Goldfarb and N. Celanovic, "Behavioral implications of piezoelectric stack actuators for control of micromanipulation," in Proc. IEEE Int. Conf. Robot. Autom., Minneapolis, MN, Apr. 1996, pp. 226-231.

[17] G. Tao and P. V. Kokotovic, "Adaptive control of plants with unknown hystereses," IEEE Trans. Autom. Control, vol. 40, no. 2, pp. 200-212, Feb. 1995

[18] K. Kuhnen, "Modeling, identification and compensation of complex hysteretic nonlinearities: A modified Prandtl-Ishlinskii approach," Eur. J. Control, vol. 9, no. 4, pp. 407-418, 2003.

[19] K. Kuhnen and H. Janocha, "Inverse feedforward controller for complex hysteretic nonlinearities in smart-material systems," Control Intell. Syst., vol. 29, pp. 74-83, 2001.

[20] U. X. Tan, T. L. Win, C. Y. Shee, and W. T. Ang, "Rate-dependent hysteresis model of piezoelectric using singularity free Prandtl-Ishlinskii model," in Proc. IEEE Int. Symp. Comput. Intell. Robot. Autom., Jacksonville, FL, Jun.2007, pp. 356-361.

[21] W. T. Ang, C. N. Riviere, and P. K. Khosla, "Feedforward controller with inverse rate-dependent model for piezoelectric actuators in trajectorytracking applications," IEEE/ASME Trans. Mechatronics, vol. 12, no. 2, pp. 1-8, Apr. 2007.

U-Xuan Tan (S'07), photograph and biography not available at the time of publication.

Win Tun Latt (S'08), photograph and biography not available at the time of publication.

Cheng Yap Shee, photograph and biography not available at the time of publication.



Cameron N. Riviere (S'94-M'95) received the B.S degree in aerospace engineering and ocean engineering from Virginia Polytechnic Institute and State University, Blacksburg, in 1989, and the Ph.D. degree in mechanical engineering from The Johns Hopkins University, Baltimore, MD, in 1995.

Since 1995, he has been with the Robotics Institute, Carnegie Mellon University, Pittsburgh, PA, where he is currently an Associate Research Professor and the Director of the Medical Instrumentation Laboratory. He is also an Adjunct Faculty Member of the Department of Rehabilitation Science and Technology, University of Pittsburgh, Pittsburgh. His current research interests include medical robotics, control systems, signal processing, learning algorithms, and biomedical applications of human-machine interfaces.

Dr. Riviere was one of the Guest Editors of the Special Issue on Medical Robotics of the PROCEEDINGS OF THE IEEE (September 2006).

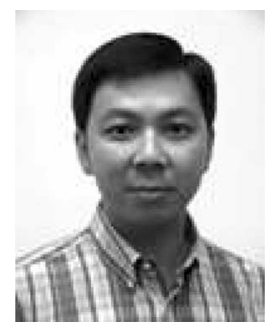

Wei Tech Ang (S'98-M'04) received the B.Eng. and M.Eng. degrees in mechanical and production engineering from Nanyang Technological University, Singapore, in 1997 and 1999, respectively, and the $\mathrm{Ph} . \mathrm{D}$. degree in robotics from Carnegie Mellon University, Pittsburgh, PA, in 2004

Since 2004, he has been an Assistant Professor in the School of Mechanical and Aerospace Engineering, Nanyang Technological University. His current research interests include sensing and sensors, actuators, medical robotics, rehabilitative and assisstive technology, mechanism design, kinematics, signal processing, and learning algorithms. 